\title{
Antiviral Effects of Pomegranate Peel Extracts on Human Norovirus in Food Models and Simulated Gastrointestinal Fluids
}

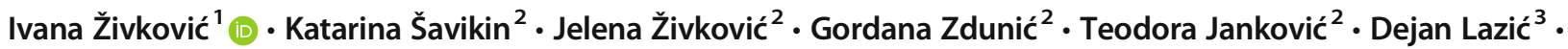 \\ Dragoslava Radin ${ }^{4}$
}

Accepted: 24 March 2021 / Published online: 7 April 2021

(C) The Author(s), under exclusive licence to Springer Science+Business Media, LLC, part of Springer Nature 2021

\begin{abstract}
Human noroviruses $(\mathrm{HuNoV})$ are the dominant cause of viral gastroenteritis in all age groups worldwide. In this study, we investigated the effects of pomegranate peel extract (PPE) on the reduction of HuNoV in different food models, on surfaces of fresh produce (green onion and cherry tomato), in low-fat milk, and simulated gastrointestinal fluids. The antiviral efficacy of PPE against HuNoV was evaluated by quantifying the number of residual virus genomes using a quantitative reverse transcription PCR (qRT-PCR) assay. Pomegranate peel, considered as a waste product of industrial processing, is known for beneficial health effects and broad antimicrobial activity due to the high content of phenolic compounds and tannins. PPE showed significant antiviral properties against HuNoV both in phosphate-buffered saline (PBS) and simulated gastric fluid. The reduction of HuNoV by pomegranate juice was lower than with PPE, which could be attributed to the lower content of antimicrobial compounds. A pretreatment of cherry tomato and green onion surfaces with PPE significantly reduced the amount of HuNoV particles that adhered to those surfaces during subsequent virus suspension treatment. A detrimental effect of PPE on HuNoV structure was confirmed by transmission electron microscopy. Our results indicate that PPE is a natural antiviral agent effective against food-borne noroviruses.
\end{abstract}

Keywords Human norovirus $\cdot$ Pomegranate peel extract $\cdot$ Antiviral effect $\cdot$ Food model

\section{Introduction}

Human noroviruses (HuNoV) are the predominant cause of viral gastroenteritis in all age groups worldwide. Using the Delphi technique, risk assessment experts throughout Europe have identified food-borne viruses (hepatitis A virus and $\mathrm{HuNoV}$ ) as food safety priorities [1]. HuNoV is the most

Ivana Živković

ivanazivkovic25@gmail.com

1 Institute for Vegetable Crops, 71 Karadjordjeva, Smederevska Palanka, Serbia

2 Institute for Medicinal Plants Research "Dr Josif Pančić", 1 Tadeuša Košćuška Street, Belgrade 11000, Serbia

3 East Diagnostics, 32 Golsvordijeva Street, Belgrade 11000, Serbia

4 Faculty of Agriculture, Institute for Food Technology and Biochemistry, University of Belgrade, 6 Nemanjina Street, Belgrade 11080, Serbia commonly reported enteric virus in food outbreaks in Europe. In the United States, it causes about 19 to 21 million cases of vomiting and/or diarrhea case per year [2]. Although potential norovirus vaccines are in development, currently, none are licensed for public use that would prevent the occurrence of diseases. Another approach to preventing food contamination is to inactivate virus particles before food consumption. The application of plant extracts, which contain several safe compounds known for their antimicrobial activity, is one way to achieve this [3]. Pomegranate (Punica granatum L.) peel, a byproduct of the juice processing industry, is known for its beneficial health effects and broad antimicrobial activity. Pomegranate peel extract (PPE) and pomegranate juice (PJ) contain high levels of ellagitannins. Punicalagin and its derivatives are major compounds likely responsible for antimicrobial and antioxidant activity [4]. Ellagitannins and anthocyanins from PPE and PJ act as prebiotics and have a synergistic effect in promoting probiotic properties. They inhibit pathogens and stimulate beneficial microbiota growth in human guts [5] and may be important natural additives for the food 
industry to improve safety, quality, and food preservation [6]. Moreover, PPE and PJ were reported to have good antibacterial [7] and antiviral activity [8]. Additionally, among 13 tested food extracts and juices, only PJ and cranberry juice reduced the specific binding ability of HuNoV P particles [9]. Until recently, HuNoV could not be grown in cell culture. Therefore, most of the research concerning the influence of different factors was conducted on its surrogates. The latest data suggests $\mathrm{HuNoV}$ is more resistant to typical food and environmental control measures than surrogate viruses, thus questioning the suitability of surrogates [10].

Considering all the beneficial properties of PPE, we set out to investigate its antiviral properties and ability to reduce HuNoV levels in different food models, on surfaces of fresh produce, in low-fat milk as representative of complex matrix and simulated gastrointestinal fluids. The antiviral properties of PPE against HuNoV were evaluated by quantifying the number of residual virus genomes using a quantitative reverse transcription PCR (qRT-PCR).

\section{Materials and Methods}

\section{Pomegranate Peel Extracts (PPE) and Pomegranate Juice (PJ)}

Wild pomegranate fruits were collected from the village Do (Bosnia and Herzegovina). The peel was separated, chopped, and dried. After a maceration of dried pomegranate peel with $70 \%$ ethanol, the extract was evaporated under low pressure at temperature $<50{ }^{\circ} \mathrm{C}$ to obtain dry extract (PPE). Commercial PJ declared as pure organic matter juice ( $\mathrm{pH}$ 3.3) was purchased from a local grocery store.

\section{Total Phenolic and Total Tannins Content}

Total phenolic content was analyzed spectrophotometrically using the slightly modified Folin-Ciocalteu method. The absorbance was measured at $765 \mathrm{~nm}$ by UV/VIS spectrophotometer (HP 8453, Agilent Technologies, USA). The calibration curve was prepared using gallic acid (GA) $(0-100 \mathrm{mg} / \mathrm{L})$. The results were expressed as milligrams of GA equivalents per gram of dry weight of the extract (mg GAE/g DW) or mg GAE/100 mL juice. The tannin content analyses were performed according to the European Pharmacopeia 7.0 using phosphomolybdotungstic reagent [11]. The absorbance of the final mixture was measured at $760 \mathrm{~nm}$ by UV/VIS spectrophotometer (HP 8453, Agilent Technologies, USA). The results were calculated and expressed as a percent of pyrogallol.

HPLC analysis was carried out on instrument Agilent 1200 RR HPLC (Waldbronn, Germany), equipped with DAD detector, using reverse-phase analytical column
Zorbax SB-C18 (Agilent), ( $5 \mu \mathrm{m}$ particle size; $150 \mathrm{~m} \times$ $4.6 \mathrm{~mm}$ i.d.). Mobile phase A was the solution of orthophosphoric acid in water $(1 \%, v / \mathrm{v})$, and mobile phase B was acetonitrile; flow rate was $1 \mathrm{~mL} / \mathrm{min}$. The injection volume was $5 \mu \mathrm{L}$, and the column temperature was maintained at $25^{\circ} \mathrm{C}$. Detection was done at 260 and $320 \mathrm{~nm}$. The amounts of investigating compounds were calculated using calibration curves of standards (punicalagin, punicalin, gallic acid, and ellagic acid).

\section{Virus Stock}

A human fecal stool sample positive for food-borne $\mathrm{HuNoV}$ GII was collected during the epidemic at University Children's Hospital - Tiršova (Belgrade, Serbia). A human fecal stool sample was dissolved (w/vol) in phosphatebuffered saline (PBS, $\mathrm{pH} 7.2$ ) to obtain $10 \%$ suspension, which was centrifuged $\left(14,000 \mathrm{G} / 5 \mathrm{~min}\right.$ at $\left.20^{\circ} \mathrm{C}\right)$ and filtrated through $0.45 \mu \mathrm{m}$ and $0.22 \mu \mathrm{m}$ pore-size filters (Thermo Fisher Scientific, Germany). For the number of genome equivalents (GE) of HuNoV in the suspension to be determined, the standard curve was prepared using positive control (PC) template from Quantification of Norovirus genotype 1 and 2, Primer DesignTM Ltd., genesig kit.

\section{HuNoV Treatment with PPE in Gastrointestinal Fluids and Milk}

The stock of HuNoV suspension was mixed in a 1:10 ratio with PBS (AppliChem, Barcelona, Spain), simulated gastric fluid (SGF; pH 1.2, USP XXII Formulation, Ricca Chemical Company, Arlington, USA), simulated intestinal fluid (SIF, pH 7.5, USP XXII Formulation, Ricca Chemical Company, Arlington, USA), low-fat milk ( $\mathrm{pH} 6.8 ; 0.5 \%$ fat, purchased at a local store). Dry PPE was dissolved in a mixture of $30 \%$ ethanol and 5\% dimethyl sulfoxide (DMSO) to obtain a stock of $10 \mathrm{mg} / \mathrm{mL}$. Subsequently, PPE was added to the mixtures to obtain a final concentration of $5 \mathrm{mg} / \mathrm{mL}$. All samples were incubated at $37^{\circ} \mathrm{C}$ for 1,2 , and $6 \mathrm{~h}$.

\section{HuNoV Treatment with PPE on the Surfaces of Fresh Vegetables}

Fresh cherry tomato and green onion, obtained from the local store, were used as representatives of fresh vegetables. After rinsing with sterile distilled deionized water, they were airdried in laminar flow for $20 \mathrm{~min}$ and exposed to UV for $15 \mathrm{~min}$. Two methods were used to investigate antiviral properties of PPE on surfaces of vegetables: (1) the suspension of HuNoV (PBS, $10^{5} \mathrm{GE} / \mu \mathrm{L}$ ) was first sprayed on the vegetable surface, and after drying at room temperature (RT), PPE was applied (5 mg/mL); and vice versa (2) first PPE was applied, 
and after drying at room temperature, HuNoV suspension was added. Samples were kept at RT for $1 \mathrm{~h}$ contact time.

\section{RNA Extraction and RT-qPCR Assay}

Norovirus RNA from PBS, SIF, SGF suspensions with/ without PPE and PJ was extracted with a Ribo-Sorb kit (Sacace Biotechnologies, Italy). Internal extraction control $(10 \mu \mathrm{L})$ was added to all samples during the isolation procedure to control the extraction process of each sample. From milk as a complex matrix, RNA was isolated by a Direct-zol ${ }^{\text {tm }}$ RNA miniPrep Plus kit (Zymo Research, USA). The extraction of total RNA from surfaces of a cherry tomato and green onion was done by the direct method with $1 \mathrm{~mL}$ of TrizolTM Reagent (Invitrogen, USA), as previously described [12]. RTqPCR assays were done using Mic Real-Time PCR Cycler (Bio Molecular Systems, Australia) and Rotavirus/ Norovirus/Astrovirus Real-TM kit (Sacace Biotechnologies, Italy), according to the manufactures instructions. The amplification reaction mixture (total $5 \mu \mathrm{L}$ per reaction) contained PCR-mix-1, PCR-mix-1/IC, RT-PCR-mix-2, hot-start Taq polymerase, M-MLV Revertase, RT-G-mix-2.

\section{Transmission Electron Microscopy Analysis}

A virus suspension (PBS, $10^{6} \mathrm{GE} / \mu \mathrm{L}$ ) was either treated with PPE $(5 \mathrm{mg} / \mathrm{mL})$ or with solvents (control) for $1 \mathrm{~h}$ at $37^{\circ} \mathrm{C}$. Subsequently, samples were fixed by the addition of glutaraldehyde to a final concentration of $2.5 \%$. Fixed samples were applied to electron microscopy grids, dried at room temperature, rinsed with distilled water, stained with $4 \%$ uranyl acetate (Ultra Stain I), and examined using an EM Stain system (Leica Microsystems, Austria). Specimens were viewed with an SM 12 transmission electron microscope (Philips/FEI, The Netherlands) at $80 \mathrm{kV}$. The digital images were obtained using the ITEM Mega View CCD.

Statistical analysis was done with SPSS software (version 23, IBM, USA). Samples (done in triplicates) were compared with one-way ANOVA and Tukey post-hoc test. Statistical significance cutoff was $p<0.05$.

\section{Results and Discussion}

Antiviral activity of PPE $(5 \mathrm{mg} / \mathrm{mL})$ against HuNoV was tested based on previous research on the evaluation of antinoroviral properties of 12 plant extracts [13]. Commercial PJ juice was used to compare antiviral activity with PPE. The stock suspension of HuNoV contained approximately $10^{6} \mathrm{GE}$ per $\mu \mathrm{L}$, as determined by absolute quantification.

\section{Chemical Composition}

Pomegranate peel is known as a rich source of phenols [14], total phenolic (320.16 mg GAE/g) and tannins (17.37 mg GAE/g) were detected in PPE used in this work, and it was higher compared to PJ (Table 1). Likewise, Hasnaoui et al. [15] and Li et al. [16] reported relatively high content of total phenolic in pomegranate peel (261.70 mg GAE/g). According to HPLC analysis, the dominant compound in PPE and PJ was punicalin (197.13 and $86.93 \mathrm{mg} / \mathrm{g}$, respectively), followed by punicalagin ( 54.23 and $43.23 \mathrm{mg} / \mathrm{g}$ ). The ellagic acid content was low in commercial PJ $(6.49 \mathrm{mg} / \mathrm{g})$, while it was higher in PPE $(25.42 \mathrm{mg} / \mathrm{g})$. Similar results were reported by Li et al. [16], who found that punicalagin and ellagic acid were among the dominant compounds of analyzed polyphenols extracted from pomegranate peel collected from different orchards in China.

In the PJ sample used in this study, made of $100 \%$ pomegranate with no other added components, the active compounds have been present in a lesser amount than in the PPE. This is in accordance with other studies that have indicated that the pomegranate peel has a higher concentration of phenolic compounds than pomegranate juice [17].

\section{Antiviral Activity of Pomegranate Peel Extract and Juice}

The HuNoV suspension in PBS was used to test the influence of PPE treatment and persistence in PJ, SGF, SIF, and milk. The initial concentration for all solutions was $5.01 \pm 0.04$ $\log _{10}$ GE. The number of HuNoV was determined by absolute quantification [18]. In previous experiments, it was confirmed that PBS had no influence on the reduction of HuNoV. The highest antiviral activity of PPE $(5 \mathrm{mg} / \mathrm{mL})$ was obtained when added to a mixture of PBS and HuNoV. The persistence of $\mathrm{HuNoV}$ after treatment with PPE was $2.60 \pm 0.04 ; 2.18 \pm$ 0.05 and $1.62 \pm 0.03 \log _{10}$ GE with contact time $1 \mathrm{~h}, 2 \mathrm{~h}$ and $6 \mathrm{~h}$ at $37^{\circ} \mathrm{C}$, respectively $(p<0.05)$ (Fig. 1a). As well, the

Table 1 Content of total phenolics (mg GAE/g DW for PPE and mg GAE/100 mL juice). Total tannins (\%), and individual polyphenolic compounds (mg-g DW) in pomegranate peel extracts (PPE) and pomegranate juice (PJ)

\begin{tabular}{lll}
\hline Compounds & PPE & PJ \\
\hline Total phenolics & $320.16 \pm 2.39$ & $146 \pm 2.01$ \\
Total tannins & $17.37 \pm 0.64$ & $5.21 \pm 0.22$ \\
Punicalagin & $54.23 \pm 1.65$ & $43.23 \pm 1.45$ \\
Punicalin & $197.13 \pm 3.48$ & $86.93 \pm 2.45$ \\
Gallic acid & $6.83 \pm 0.53$ & $6.69 \pm 0.33$ \\
Ellagic acid & $25.42 \pm 0.80$ & $6.49 \pm 0.71$ \\
\hline
\end{tabular}


antinorovirus effect of PPE was assessed by quantifying the percentage of norovirus genome remaining after treatment (Fig. 1b) [18]. These results indicate the ability of PPE to reduce $\mathrm{HuNoV}$ by $99 \%$ in PBS. To elucidate the mechanisms of action of PPE, we examined the human norovirus particles in PBS (concentration $5.01 \pm 0.04 \log _{10} \mathrm{GE} / \mu \mathrm{L}$ ) (Fig. 2a) before and after treatment with TEM. After treatment with PPE $(5 \mathrm{mg} / \mathrm{mL}$ ), the capsid proteins of $\mathrm{HuNoV}$ had damage and disruption (Fig. 2b). Changes in the morphology of NoV GII4-like particles treated with grape seeds [19] and in the morphology of FCV and MNV treated with blueberry proanthocyanidins [20] observed with TEM were similar to structure changes HuNoV with PPE. However, this mechanism is not yet fully understood and requires further and more detailed investigation. Similar antiviral activity for NoV GII.4 in PBS by grape seeds has been published for extract and homogenized lettuce extract [19]. Treatment with an extract of persimmon fruit containing $0.96 \mathrm{mg} / \mathrm{mL}$ tannin caused a human norovirus genome reduction of more than $90 \%$ [18]. In low-fat milk, PPE showed antiviral activity, but reductions of HuNoV were decreased compared to PBS. The presence of $\mathrm{HuNoV}$ in milk after treatment with PPE were $3.34 \pm 0.08$, $3.07 \pm 0.04$ and $2.54 \pm 0.1$ the $\log _{10}$ GE after 1,2 and $6 \mathrm{~h}$, respectively $(p<0.05)$ (Fig. 1a). The chemical content of milk (fat and protein) has a protective effect on $\mathrm{HuNoV}$, and this has resulted in less reduction in the norovirus genome compared to PBS, about 20\% ( $p<0.05)$ (Fig. 1a and b). These results indicate that PPE denatures viral capsid in complex food matrix more than $91 \%$ (Fig. 1b) [21]. For examining the antiviral properties of PPE, it is important to study their effect under the conditions that will occur during consumption. For the transit time of orally consumed substances in the corresponding gastrointestinal segments to be stimulated, 2 and $6 \mathrm{~h}$ contact times of HuNoV and PPE for the gastric (stomach) and intestinal (small intestines) simulation were selected, respectively [22]. The mixture of HuNoV in simulated gastric $(\mathrm{pH}$ 1.3) and intestinal $(\mathrm{pH} 7.5)$ fluid did not cause a significant reduction of $\mathrm{HuNoV}$ after 2 and $6 \mathrm{~h}$ (Fig. 1a). However, the persistence of $\mathrm{HuNoV}$ in SGF after antiviral effects of PPE was $2.7 \pm 0.09 \log _{10}$ GE after $2 \mathrm{~h}$. A low $\mathrm{pH}$ caused the decline of antiviral activity of PPE after $6 \mathrm{~h}$. The presence of HuNoV (SGF, $6 \mathrm{~h}$ ) was higher than after $2 \mathrm{~h}$ and amounted to $3.41 \pm 0.06$. The decrease in the antiviral activity of PPE in SGF after $6 \mathrm{~h}$ could be attributed to the chemical degradation of the gastrointestinal fluid itself [22]. The number of HuNoV in SIF decreased and after 2 and $6 \mathrm{~h}$ was $3.61 \pm 0.09$ and $2.24 \pm 0.02$, respectively $(p<0.05)$ (Fig. 1a). The effect of PPE in SGF (2 h) and SIF (6 h) was 99\% (Fig. 1b), which means that a residual percentage of norovirus genome after treatment was 0.8 and $0.3 \%$ (Fig. 1c). The persistence of HuNoV in PJ after 1, 2 and $6 \mathrm{~h}$ was $3.28 \pm 0.06,3.09 \pm 0.09$ and $2.73 \pm 0.04 \log _{10}$, respectively $(p<0.05)$ (Fig. 1a and c). In our study, the $\mathrm{PJ}(\mathrm{pH} 3.3)$ reduced norovirus presence by $97 \%(1 \mathrm{~h})$ and $99 \%(2 \mathrm{~h}$ and 6 h) (Fig. 1b). Obtained reduction by PJ was lower than with PPE, which could be connected with the lower content of dominant antimicrobial compounds. Potential inhibitors and immune-boosting functional food ingredients are crucial in preventing pandemic diseases such as Covid-19 [23]. A recent study showed that polyphenols from PPE inhibit and reduce viral protein activity and can be used to treat SARS-CoV-2 infections [24]. Since one of the symptoms of Covid-19 infection and HuNoV is diarrhea, using PPE could be a significant preventive treatment.

\section{Antiviral Properties of PPE on Surfaces of Fresh Vegetables}

The possibility of HuNoV reduction on cherry tomato and green onion surfaces is important since they present food models that dominate in salads, sandwiches, buffet meals, and ready-made food. The reduction of $\mathrm{HuNoV}$ on fresh vegetables depended significantly on the manner of application of PPE and HuNoV suspension [20]. The initial concentration of $\mathrm{HuNoV}$ was $5 \log _{10} \mathrm{GE} / \mathrm{uL}$ and served as the control. When method 1 was used, the residual virus particles after PPE treatment were $2.58 \pm 0.08$ and $2.49 \pm 0.15 \operatorname{logs}_{10}$ GE on cherry tomatoes and green onions, respectively $(p<0.05)$. Better reduction was obtained when method 2 was used. Residual virus particles after PPE treatment were $1.18 \pm 0.06$ and 1.30 $\pm 0.12 \operatorname{logs}_{10}$ GE on cherry tomatoes and green onions $\log s_{10}$ GE, respectively $(p<0.05)$ (Fig. 1d).

To conclude, a significantly higher reduction of $\mathrm{HuNoV}$ particles was acquired if fresh vegetables were first treated with PPE and afterward with virus suspension. Usually, fresh products do not receive an appropriate treatment to reduce pathogenic bacteria or viruses to reach a safe level. Accordingly, implementing a strategy that implies adding PPE is likely to reduce HuNoV on surfaces of fresh products which would increase their safety.

\section{Conclusion}

With the frequently reported food-borne HuNoV outbreaks, the use of antiviral compounds originating from natural sources is becoming increasingly relevant. It is believed that disabling the viral attachment to the host cell may be achieved by damaging the capsid of the virion or altering the receptor on the cell membrane. This study shows that PPE and PJ had a significant effect on HuNoV reduction. This suggests a potential application of PPE as natural antiviral agents and condiments in the control of virus contamination of food during its production and processing. In this respect, the ability of PPE to reduce $\mathrm{HuNoV}$ by more than $98.5 \%$ on the surfaces of cherry tomato and green onion is important since these 

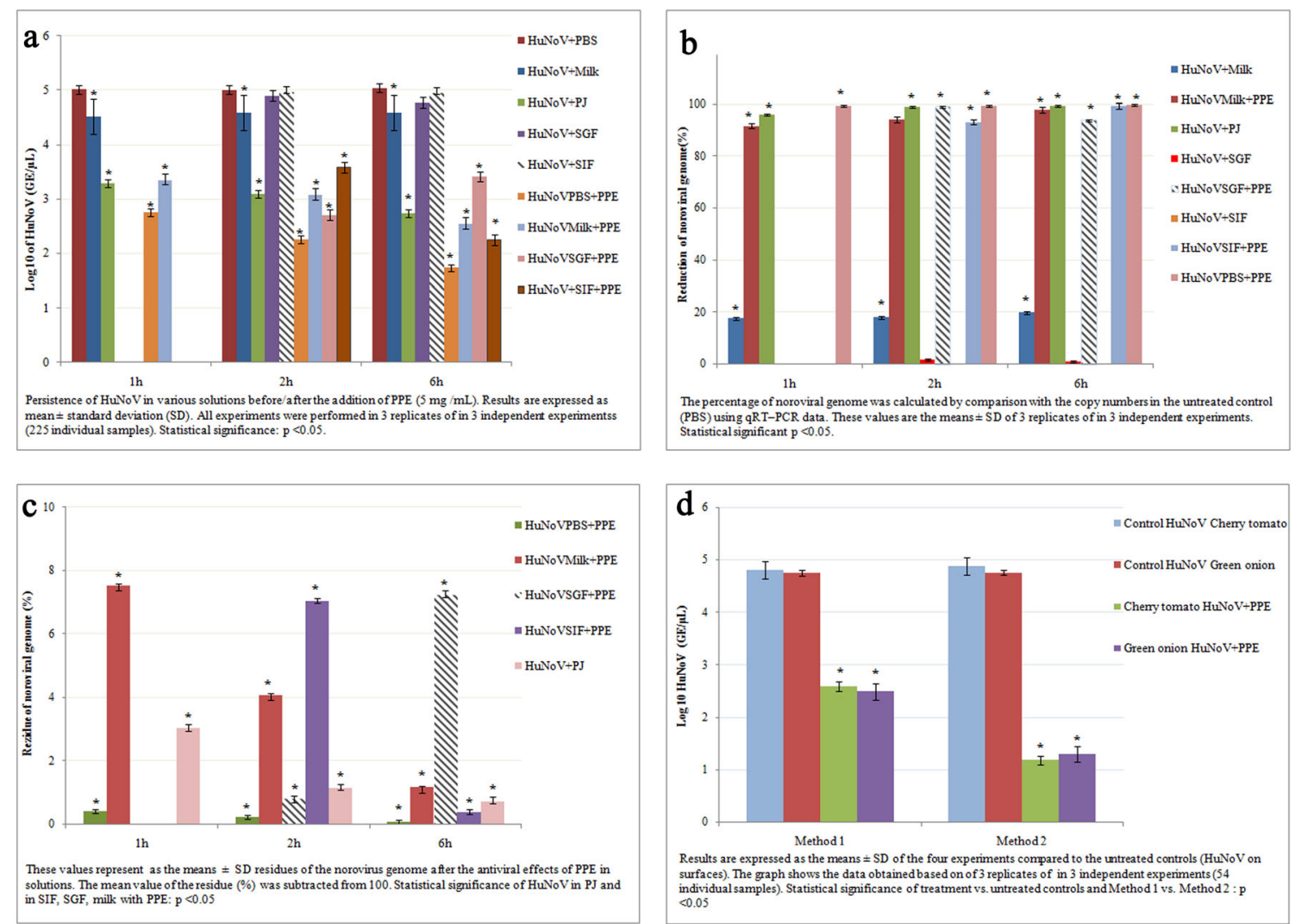

Fig. 1 a Persistence of human norovirus (initial concentration $5.01 \pm 0.04$ $\log _{10} \mathrm{GE} / \mu \mathrm{L}$ ) in phosphate buffer (PBS), simulated gastric (SGF, pH 1.3) and intestinal (SIF, pH 7.5), pomegranate juice (PJ), food model (low-fat milk, $\mathrm{pH}$ 6.8) and after treatment with pomegranate peel extract (PPE, $5 \mathrm{mg} / \mathrm{mL}$ ). All experiments were performed in three replicates of in three independent experimentss (225 individual samples). Statistical significance: $p<0.05$. b Antiviral effects of pomegranate peel extract $(5 \mathrm{mg} / \mathrm{mL})$. The percentage reduction of norovirus genome (initial concentration $\left.5.01 \pm 0.04 \log _{10} \mathrm{GE} / \mu \mathrm{L}\right)$ after treatment with PPE was

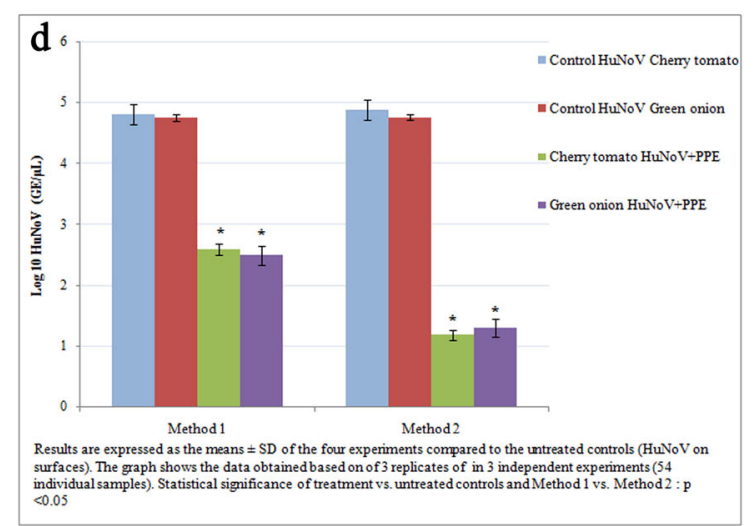

calculated by comparing the numbers of HuNoV genome in the untreated control (PBS, SGF, SIF, Milk) using qRT-PCR data. Statistical significance: $p<0.05 \mathrm{c}$ The residual norovirus genome after treatment with PPE $(5 \mathrm{mg} / \mathrm{mL})$ in SIF, SGF, milk + PPE vs. HuNoV (PBS) (under 10\%): $p<0.05$. The percentage of residual norovirus genome was subtracted from 100. d Antiviral effects of PPE on HuNoV on surfaces of fresh vegetables. Statistical significance of treatments vs. untreated controls: $p<0.05$

Fig. 2 Transmission electron microscopy analysis of human norovirus (concentration $5.01 \pm$ 0.04). a HuNoV in PBS (untreated control). b HuNoV after treatment with PPE (5 mg/ $\mathrm{mL}$ )

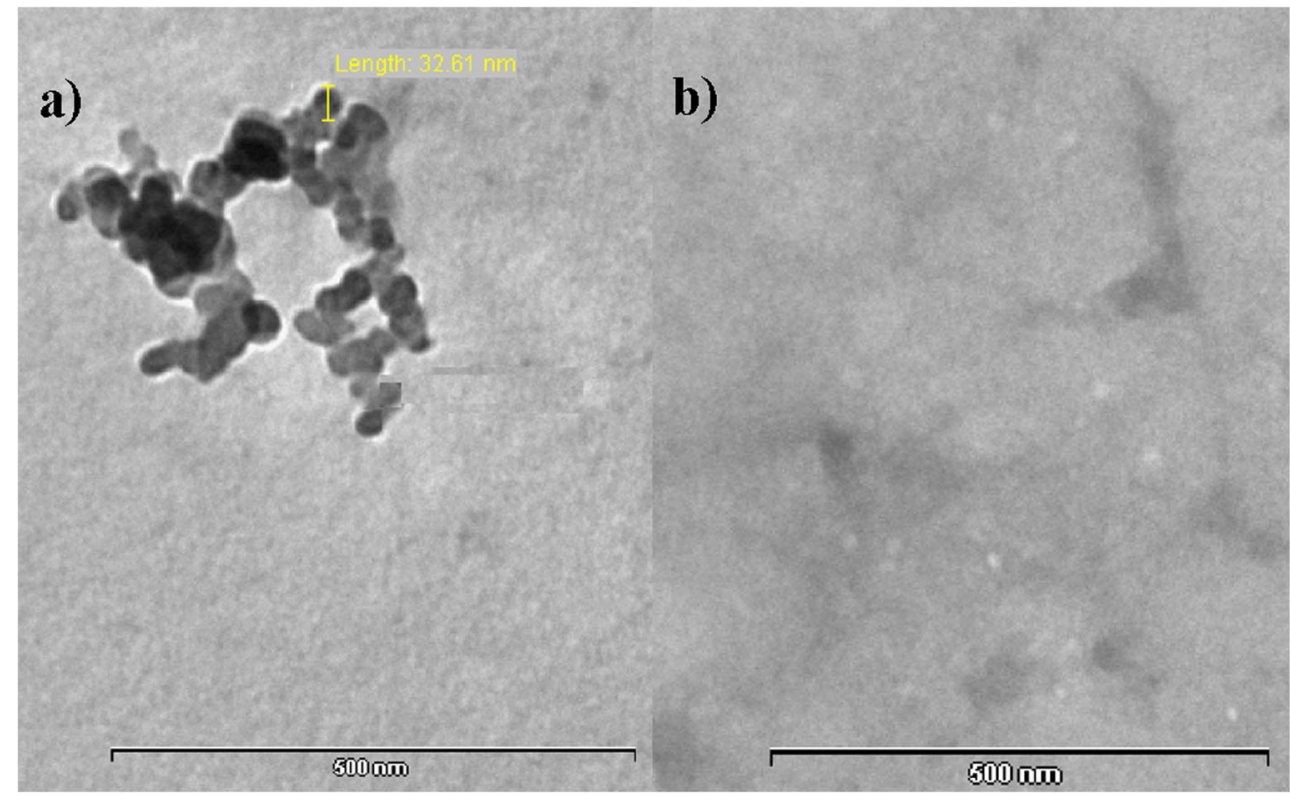


products are often present in salads, sandwiches, buffet meals, ready-to-eat food. These types of foods rarely undergo any heat processing prior to consumption, facilitating easy virus transmission. The results showed that the method of application is important for the antiviral activity of PPE. The antiviral activity of PPE in the gastrointestinal environment allows potential treatment against human norovirus, which requires further supporting clinical trial data. Overall, the progress towards the development of natural antiviral agents that can be applied or consumed is of great importance, as they can usually be produced with less financial investment, and their natural origin provides a good perception of the consumer.

Abbreviations $G A E / g D W$, Milligrams of gallic acid equivalents per gram of dry weight of the extract; GE, Genome equivalents; HuNoV, Human norovirus; $P B S$, Phosphate buffered saline; $P J$, Pomegranate juice; $P P E$, Pomegranate peel extract; $S G F$, Simulated gastric fluid; $S I F$, Simulated intestinal fluid; TEM, Transmission electron microscopy

Acknowledgements This research was supported by the Ministry of Education, Science and Technological Development of the Republic of Serbia, contract numbers 451-03-68/2020-14/200116 and 451-03-68/ 2020-14/200003.

Contributions All authors contributed to the performance and writing of the paper.

\section{Declarations}

Conflict of Interest The authors declare no conflict of interest.

\section{References}

1. Rowe G, Bolger F (2016) Final report on 'the identification of food safety priorities using the Delphi technique'. EFSA Support Publ 13:1-139 https://efsa.onlinelibrary.wiley.com/doi/epdf/10.2903/sp. efsa.2016.EN-1007

2. Bartsch SM, O'Shea KJ, Lee BY (2020) The clinical and economic burden of norovirus gastroenteritis in the United States. J Infect Dis 222:1910-1919. https://doi.org/10.1093/infdis/jiaa292

3. Li D, Baert L, Uyttendaele M (2013) Inactivation of food-borne viruses using natural biochemical substances. Food Microbiol 35: 1-9. https://doi.org/10.1016/j.fm.2013.02.009

4. Bialonska D, Kasimsetty SG, Schrader KK, Ferreira D (2009) The effect of pomegranate (Punica granatum L.) byproducts and ellagitannins on the growth of human gut bacteria. J Agric Food Chem 57:8344-8349. https://doi.org/10.1021/j901931b

5. Ismail T, Akhtar S, Riaz M, Ismail A (2014) Effect of pomegranate peel supplementation on nutritional, organoleptic and stability properties of cookies. Int J Food Sci Nutr 65:661-666. https://doi. org/10.3109/09637486.2014.908170

6. Nair M, Saxena A, Kaur C (2018) Effect of chitosan and alginate based coatings enriched with pomegranate peel extract to extend the postharvest quality of guava (Psidium guajava L.). Food Chem 240:245-252. https://doi.org/10.1016/j.foodchem.2017.07.122

7. Nozohour Y, Golmohammadi R, Mirnejad R, Fartashvand M (2018) Antibacterial activity of pomegranate (Punica granatum L.) seed and peel alcoholic extracts on Staphylococcus aureus and Pseudomonas aeruginosa isolated from health centers. J Appl Biotechnol Rep 5:32-36. https://doi.org/10.29252/jabr.01.01.06

8. Moradi MT, Karimi A, Rafieian-kopaei M, Rabiei-Faradonbeh M, Momtaz H (2020) Pomegranate peel extract inhibits internalization and replication of the influenza virus: an in vitro study. Avicenna $\mathrm{J}$ Phytomed 10:143-151 PMID:32257886

9. Li D, Baert L, Xia M, Zhong W, Jiang X, Uyttendaele M (2012) Effects of a variety of food extracts and juices on the specific binding ability of norovirus GII.4 P particles. J Food Prot 75:1350 1354. https://doi.org/10.4315/0362-028X.JFP-12-002

10. Knight A, Haines J, Stals A, Li D, Uyttendaele M, Knight A, Jaykus LA (2016) A systematic review of human norovirus survival reveals a greater persistence of human norovirus RT-qPCR signals compared to those of cultivable surrogate viruses. Int J Food Microbiol 216:40-49. https://doi.org/10.1016/j.ijfoodmicro.2015. 08.015

11. European Pharmacopoeia 8 (2014) Monographs on herbal drugs and herbal drug preparations. Council of Europe, Strasbourg

12. Radin D, D'Souza DH (2011) Simple and rapid detection of human Norovirus from produce using SYBR green I-based real-time RTPCR. Food Environ Virol 3:121-129. https://doi.org/10.1007/ s12560-011-9066-5

13. Živković I, Šavikin K, Zdunić G, Živković J, Bigović D, Menković N, Radin D (2018) Antiviral activity of medicinal plants extracts against food-borne norovirus. Lekovite Sirovine 38:1-4. https://doi. org 1 10.5937/leksir1838031Z

14. Gullon B, Pintado ME, Pérez-Álvarez J, Viuda-Martos M (2016) Assessment of polyphenolic profile and antibacterial activity of pomegranate peel (Punica granatum) flour obtained from coproduct of juice extraction. Food Control 59:94-98. https://doi. org/10.1016/j.foodcont.2015.05.025

15. Hasnaoui N, Wathelet B, Jiménez-Araujo A (2014) Valorization of pomegranate peel from 12 cultivars: dietary fibre composition, antioxidant capacity and functional properties. Food Chem 160:196203. https://doi.org/10.1016/j.foodchem.2014.03.089

16. Li J, He X, Li M, Zhao W, Liu L, Kong X (2015) Chemical fingerprint and quantitative analysis for quality control of polyphenols extracted from pomegranate peel by HPLC. Food Chem 176:711. https://doi.org/10.1016/j.foodchem.2014.12.040

17. Russo M, Fanali C, Tripodo G, Dugo P, Muleo R, Dugo L et al (2018) Analysis of phenolic compounds in different parts of pomegranate (Punica granatum) fruit by HPLC-PDA-ESI/MS and evaluation of their antioxidant activity: application to different Italian varieties. Anal Bioanal Chem 410:3507-3520. https://doi.org/10. 1007/s00216-018-0854-8

18. Kamimoto M, Nakai Y, Tsuji T, Shimamoto T, Shimamoto T (2014) Antiviral effects of persimmon extract on human norovirus and its surrogate, bacteriophage MS2. J Food Sci 79:M941-M946. https://doi.org/10.1111/1750-3841.12462

19. Li D, Baert L, Zhang D, Xia M, Zhong W, Van Coillie E, Jiang X, Uyttendaele M (2012) Effect of grape seed extract on human norovirus GII.4 and murine norovirus 1 in viral suspensions, on stainless steel discs, and in lettuce wash water. Appl Environ Microbiol 78:7572-7578. https://doi.org/10.1128/AEM.01987-12

20. Joshi S, Howell AB, D'Souza DH (2017) Blueberry proanthocyanidins against human norovirus surrogates in model foods and under simulated gastric conditions. Food Microbiol 63: 263-267. https://doi.org/10.1016/j.fm.2016.11.024

21. Higginbotham KL, Burris KP, Zivanovic S, Davidson P, Stewart C (2014) Antimicrobial activity of Hibiscus sabdariffa aqueous extracts against Escherichia coli O157:H7 and Staphylococcus aureus in a microbiological medium and milk of various fat concentrations. J Food Prot 77:262-268. https://doi.org/10.4315/0362028x.jfp-13-313

22. Vermaak I, Viljoen AM, Hamman JH, Van Vuuren SF (2009) The effect of simulated gastrointestinal conditions on the antimicrobial 
activity and chemical composition of indigenous south African plant extracts. S Afr J Bot 75:594-599. https://doi.org/10.1016/j. sajb.2009.02.180

23. Singh P, Tripathi MK, Yasir M, Khare R, Tripathi MK, Shrivastava R (2020) Potential inhibitors for SARS-CoV-2 and functional food components as nutritional supplement for COVID-19: a review. Plant Foods Hum Nutr 75:458-466. https://doi.org/10.1007/ s11130-020-00861-9
24. Suručić R, Tubić B, Stojiljković MP, Djuric DM, Travar M, Grabež M, Šavikin K, Škrbić R (2021) Computational study of pomegranate peel extract polyphenols as potential inhibitors of SARS-CoV-2 virus internalization. Mol Cell Biochem 476:1179-1193. https:// doi.org/10.1007/s11010-020-03981-7

Publisher's Note Springer Nature remains neutral with regard to jurisdictional claims in published maps and institutional affiliations. 